\title{
KAtex TEST FOR THE DETECTION OF URINARY ANTIGENS IN VISCERAL LEISHMANIASIS PATIENTS OF NEPAL
}

\author{
Sherchand J B*, Shah S N , Hommel M
}

\section{ABSTRACT}

The rapid detection of parasitic antigens in body fluid including urine by immunological tests such as KAtex has been a valuable addition to clinical medicine. Existing method of microscopic bone-marrow examination and immunological test for antigen -enzyme-linked immunosorbent assay (ELISA) test for visceral leishmaniasis antigen are difficult to perform in the field, and this has limited use in rural areas of endemic countries like Nepal. In this study we have tested 276 urine samples from visceral leishmaniasis patients from different endemic areas of Nepal. Sixty-seven patients $(\mathbf{2 4 . 3 \%}$ ) were found KAtex positive. The clinical picture of these patients was compared with the KAtex test and additional laboratory findings, knowledge, attitude and behaviour were assessed. High rate of KAtex positive $(97.4 \%)$ was found in bone marrow confirmed patients with high degrees of sensitivity and specificity. Hence the study confirmed that the KAtex test is suitable for the confirmation of suspected cases in the field and hospital where bonemarrow facilities are limited or where there is a lack of trained manpower as well as microscopic diagnosis is not available.

Key Words: Rapid diagnostic test, urinary antigen, visceral leishmaniasis, Nepal.

\section{INTRODUCTION}

Visceral leishmaniasis (VL) is a disease caused by protozoan parasites of the genus Leishmania, transmitted by a phlebotomine sandfly. In Indian sub-continent including Nepal, the disease is called Kala-azar. Essentially a tropical disease, VL occurs in South and Central America, in Africa and on the Indian subcontinent, but it is also endemic in Southern Europe. Overall, it has been estimated that there are 12-13 million of cases of leishmaniasis worldwide ( $90 \%$ of which occur in India, Bangladesh, Nepal and Sudan), with as many as 100000 deaths every year. ${ }^{1}$ This only represents the 'tip of the iceberg' since not all infected individuals develop a disease. Malnutrition, stress and immune defects are severity factors and it is not surprising that epidemics of VL are often associated with poverty, famine, war and immunosuppression. For instance, a massive epidemic of VL has occurred in recent years in the war zones of Southern Sudan, where about 100,000 people died of leishmaniasis since 1984 . $^{2}$ The HIV epidemic has had a major impact on the prevalence of VL in areas where the two diseases are co-endemic; in Southern Europe AIDS patients have been reported to have a 100-times greater risk of developing VL than the non-HIV population.

In Nepal, kala azar was first identified in the southeastern Terai region in 1980 and was believed to be an extension from India across the borders of Bihar province through population movement. ${ }^{3}$ The number of cases has been steadily increasing since 1980 and occasionally causing panic due to the high fatality rate. The disease has now spread to twelve districts namely Jhapa, Morang, Sunsari, Saptari, Siraha, Udayapur, Dhanusha, Mahottari, Sarlahi, Rautahat, Bara and Parsa of

* Tribhuvan University Teaching Hospital, Institute of Medicine, Maharajgunj, Kathmandu, Nepal.

** Liverpool School of Tropical Medicine, L3 5QA, Liverpool UK.

Address for correspondence : Dr. Jeevan B. Sherchand

Tribhuvan University Institute of Medicine

Parasitology and Health Research Laboratory, Kathmandu, Nepal

Email: itdre@healthnet.org.np 
eastern and central Terai regions bordering with Bihar state of India. However some cases have also been sporadically reported from western Terai region. A total of 5.5 million people live in these 12 kala-azar affected districts, eleven of which border with Bihar State of India. ${ }^{4}$ On the basis of kalaazar situation reported by HMG-EDCD Annual report, 2000, a total of 15,391 cases and 386 deaths were reported between 1980 and $1999 .{ }^{4}$ The case fatality rate (CFR) for this period varied from $0.23 \%$ to $13.2 \%$. Although the disease affects all age groups, the concentration of cases is highest amongst the 10-20 year age group. The prevalence of cases is higher in males than females. In Nepal, the published data only include recorded cases and deaths in the governmental health facilities, which means that actual kala-azar cases and deaths could be still higher.

A few entomological studies on vectors of kala-azar have been carried out. ${ }^{5}$ Phlebotomus argentipes was incriminated as the main vector of kala-azar in Nepal. The vector prevalence and density patterns are associated with the occurrence of the disease during the months of April to September (with a peak during May to August). No animal reservoir has yet been identified in Nepal.

\section{RATIONALE OF THE STUDY}

Visceral leishmaniasis is a severe disease, whose clinical symptoms are not characteristic, the diagnosis of which relies on serology and the finding of parasites in bone marrow biopsies or spleen aspirates. In many countries where VL is endemic, serology is not available and when microscopy is done, it is frequently unreliable and inherently of limited sensitivity. A simple procedure for the detection of antigen in the urine, which is easy to perform and interpret, uses robust reagents and does not require specialized equipment would be a substantial improvement in the management of $\mathrm{VL}$ in comparison with a variety of diagnostic procedures in use in Nepal.

The objective of the study was to perform a field evaluation of a new latex agglutination assay (KAtex) designed to detect leishmanial antigen in the urine of patients with VL in comparisons with variety of diagnostic procedures in use in Nepal.

\section{MATERIALS AND METHODS}

The study was a part of a preliminary multi-centre trial of urine antigen detection test for visceral leishmaniasis designed by Liverpool School of Tropical Medicine, supported by the World Health Organization (WHO) and approved by the Ethical Committee of Tribhuvan University Institute of Medicine with authority granted from Nepal Health Research Council (NHRC).

The study was conducted between September 2000 and May 2002. Patients over five years of age, having presented at the outpatient clinic of one of four different hospitals (Mahottari, Dhanusha, Siraha or Kathmandu) with suspected kala-azar, were included in the study. All these patients had a history of fever for more than 2 weeks and had a palpable splenomegaly. Urine samples were collected solely as required to confirm or exclude the presence of kala-azar. Control samples were collected from 45 healthy individuals, with no recent history of fever (15 samples each from Mahottari, Dhanusha and Siraha districts) and from 16 healthy medical students from Kathmandu. All urine samples were preserved by addition of sodium azide to a final concentration of $0.1 \%$ (wt/vol) and were stored at $4^{\circ} \mathrm{C}$ until testing (maximum storage before testing, 72h). Bone marrow biopsies were performed whenever possible, using routine procedures such as sternal and iliac crest puncture. Bone marrow slides were made using the marrow specimen, which were dried and fixed in methanol, then stained with Giemsa stain. A trained pathologist examined the stained slides microscopically. Aldehyde tests are routinely used for the diagnosis of VL in district laboratories and trained technicians performed all tests according to established procedures: $1-2 \mathrm{ml}$ of serum was placed in a clean glass tube and 1-2 drops of $40 \%$ formalin were added. The test was considered positive when milky-white jellification of the serum occurred after 2-20 minutes. Results of the aldehyde tests are available for 25 of the 276 patients in our study. Ministry of Health, Epidemiology and Disease Control Division have supplied K39 kits to certain districts; the test was performed following manufacturer's instructions. Results of K39 tests are available for 50 of the 276 patients in our study. KAtex was performed following manufacturer ${ }^{1} \mathrm{~s}$ instructions (Kalon Biological Ltd, UK). Urine samples collected in sterile containers were heated in a boiling water bath for 5 minutes before test were performed. $40 \mu \mathrm{l}$ of thoroughly mixed latex suspension was added to an equal volume of urine sample on the well of the black ceramic slide provided. KAtex and urine samples were mixed thoroughly until they were completely homogenized and covered the well up to its edges. Any agglutination, which occurred within 2 minutes, was considered positive; the test was repeated when a weak reaction was observed.

\section{RESULTS}

A total of 276 urine samples collected from different areas from patients suspected of having visceral leishmaniasis was tested by KAtex. Overall, 67 (24.3\%) were positive by KAtest (Table I). The study analysis revealed that, in the 39 patients 
for whom a bone marrow examination had been performed, KAtest was positive in 38 cases $(97 \%)$, while the positivity rate was much lower in patients where clinical suspicion was not confirmed by microscopy. The comparison of bone-marrow examination and KAtex test is shown in Table I.

\section{KAtex TEST IN DIFFERENT AGE GROUP}

The highest positivity rate of visceral leishmaniasis was found in age group between 11 and 20 (38.4\%). However, there was no significance different in all age group $(\mathrm{P}<0.05)$, but the lowest positivity rate was found in the age group between 31 and 40 . 